\title{
Constraints on Dynamical Dark Energy with Precision Cosmological Data
}

\author{
Erick Almaraz*, Axel de la Macorra\# \\ Instituto de Física, Universidad Nacional Autónoma de México, Ciudad de México, México \\ Email: *ealmaraz@estudiantes.fisica.unam.mx, "macorra@_sica.unam.mx, "
}

How to cite this paper: Almaraz, E. and de la Macorra, A. (2018) Constraints on Dynamical Dark Energy with Precision Cosmological Data. Journal of Modern Physics, 9, 302-313.

https://doi.org/10.4236/jmp.2018.92021

Received: December 26, 2017

Accepted: January 26, 2018

Published: January 29, 2018

Copyright $\odot 2018$ by authors and Scientific Research Publishing Inc. This work is licensed under the Creative Commons Attribution International License (CC BY 4.0).

http://creativecommons.org/licenses/by/4.0/

(c) (i) Open Access

\begin{abstract}
We present a Dark Energy (DE) model based on a scalar field with an inverse power law potential (IPL) $V(\phi)=M^{4+n} \phi^{-n}$. We consider three different models $n=1 / 2, n=3 / 4$ and $n=1$ and we vary the value of $M$ and the initial amount of energy density $\Omega_{\phi}$ at the scale factor $a_{c}$. We obtain a time dependent equation of state $(\mathrm{E} O S) w_{\phi}=p_{\phi} / \rho_{\phi}$, with $w_{\phi}=1 / 3$ at early times for a scale factor $a<a_{c}$ with a steep transition to $w_{\phi}=1$ at $a_{c}=1.79 \times 10^{-5}$, $9.3 \times 10^{-7}, 5.48 \times 10^{-8}$ lasting a long period of time and a subsequent descent to $w_{\phi}=-1$ for $a \sim 10^{-3}$ to finally grow to $w_{\phi}=-0.906, w_{\phi}=-0.932$, $w_{\phi}=-0.924$ for $n=1 / 2, n=3 / 4$, and $n=1$ respectively. The values of $M$ and $\Omega_{\phi}\left(a_{c}\right)$ are $M(\mathrm{eV})=4.63,127.31,2465.46$ and $\Omega_{\phi}\left(a_{c}\right)=0.038,0.148$, 0.227 for $n=1 / 2, n=3 / 4$ and $n=1$ respectively. We show the differences in the evolution of $H$, the CMB and Matter power spectra, and the redshift space distortion (RSD) $f \sigma_{8}$ parameter. Precision cosmological data allow us to test the dynamics of Dark Energy and we obtain in all three cases a reduction of $\chi_{B A O}^{2} \simeq 20 \%$ compared to $\Lambda \mathrm{CDM}$ with and an equivalent fit for CMB and SNIa data.
\end{abstract}

\section{Keywords}

Cosmology, Dark Energy, Scalar Fields

\section{Introduction}

It has passed almost twenty years since the accelerated expansion of the universe was first observed by distance measurements using type Ia supernovae (SNeIa) [1] [2]. Since that time, the acceleration of the universe has been firmly established by a series of telescopes and satellites which gather information from 
other cosmological probes such as the Cosmic Microwave Background Radiation (CMB) [3], Baryon Acoustic Oscillations (BAO) and Large Scale Structure (LSS) surveys [4] [5] [6]. In the standard paradigm of general relativity, the physical agent causing the late-time acceleration of the universe is referred to as the Dark Energy (DE). To elucidate the nature of the $\mathrm{DE}$ is one of the main goals of modern cosmology. The amount and precision of observational data collected in the last decade have grown up impressively. It is now possible to test and discriminate different DE models and projects scheduled to start operating in the near future such as DESI [7], LSST [8], and Euclid [9] will provide conclusive evidence on the subject.

In the standard cosmological model $(\Lambda \mathrm{CDM})$, the energy density of the universe at present day is made up of $69 \%$ of DE described by a cosmological constant $\Lambda, 26 \%$ of dark matter (DM) while only $5 \%$ corresponds to the Standard Model (SM) particles consisting mainly of photons, neutrinos, and ordinary matter (protons, neutrons, and electrons). However, although the $\Lambda \mathrm{CDM}$ model has proved to agree very well with the observations [3], there is no understanding of the physical mechanism that determines the origin and the magnitude of the cosmological constant $\Lambda$ and hence of why and when the universe accelerates [10]. This lack of understanding is commonly expressed in terms of the "fine-tuning" and the "coincidence" problems; the former asks why the observations set the value of the DE density $\rho_{\Lambda}$ to almost $10^{120}$ orders of magnitude below conservative estimations based on quantum field theory, while the "coincidence" problem inquires why the energy densities of the DE and the matter are of the same order of magnitude precisely at present time. This scenario has enforced the quest of other mechanisms to explain the nature of DE. Alternative to $\Lambda$, scalar fields $\phi$ have been extensively explored as a source of DE [11]-[16] because of their intrinsic link with the Standard Model of particle physics and its extensions, opening up the possibility to explain the nature of DE from first principles. In this paper we study the constraints set by recent cosmological observations on a scalar field model with inverse power law (IPL) potential $V=M^{4+n} \phi^{-n}$ giving a good fit to cosmological observations. The IPL potentials may arise from the non-perturbative dynamics of a non-abelian gauge group $S U\left(N_{c}\right)$ with $N_{c}$ colors and $N_{f}$ massless fields [17]-[23]. Unlike ordinary quintessence where the scalar field representing the $\mathrm{DE}$ is subdominant until recent times, our model considers the possibility that this component may play also an important role in the early universe, leading to cosmological imprints that can be tested against current observational information. However, here we are more interested in constraining IPL potentials with recent precision cosmological data than in a theoretical derivation of the IPL potential.

The outline of the paper is the following. In Section 2 we discuss the basic picture of the model and present the dynamical equations. The constraints set by the data are presented in Section 3. We study the cosmological consequences of the model in Section 4 and give our conclusions in Section 5. 


\section{Dynamical Dark Energy}

Scalar field $\phi$ theories have been proposed as possible sources to describe DE and a wide range of models have been studied in recent years [11]-[16] [24]. Particularly, IPL potentials

$$
V(\phi)=M^{4+n} \phi^{-n}
$$

proposed by RP [25] [26] [27] have been widely investigated giving an equivalent fit as $\Lambda \mathrm{CDM}$ [28] [29] [30]. The evolution of the energy density $\rho_{\phi}=\dot{\phi}^{2} / 2+V(\phi)$, pressure $p_{\phi}=\dot{\phi}^{2} / 2-V(\phi)$ and equation of state (EoS) $w_{\phi}=p_{\phi} / \rho_{\phi}$ depends on the parameters $n, M$ (with mass dimensions) and the initial conditions of $\phi$. All these three parameters have an important impact in determining the size and slope of $w_{\phi}$ at present time and must be adjusted by the cosmological observations [15] [16] [28] [29] [30]. These quantities are free parameters to be adjusted by the cosmological observations or the choice of model.

The evolution of $\phi$ in a homogeneous flat universe described by the Friedmann-Lemaitre-Robertson-Walker metric is completely determined by the Klein-Gordon equation

$$
\ddot{\phi}+3 H \dot{\phi}+\frac{\mathrm{d} V}{\mathrm{~d} \phi}=0,
$$

where the dots stand for cosmic time derivatives, and the Hubble expansion rate:

$$
H^{2} \equiv\left(\frac{\dot{a}}{a}\right)^{2}=\frac{8 \pi G}{3} \rho_{\text {tot }}=\frac{8 \pi G}{3}\left(\rho_{m o} a^{-3}+\rho_{r o} a^{-4}+\rho_{\phi}\right)
$$

with $\rho_{\text {tot }}(a)=\rho_{\text {mo }} a^{-3}+\rho_{r o} a^{-4}+\rho_{\phi}$ the total energy density, $\rho_{m o}, \rho_{r o}$ the present day energy densities of matter and radiation and the redshift $z$ is given by $a=(1+z)^{-1}$ with $a_{o}=1$. The energy density and pressure for the scalar field $\phi$ are

$$
\rho_{\phi}=\frac{1}{2} \dot{\phi}^{2}+V(\phi), \quad p_{\phi}=\frac{1}{2} \dot{\phi}^{2}-V(\phi),
$$

with an equation of state (EOS)

$$
w_{\phi}=\frac{p_{\phi}}{\rho_{\phi}}=\frac{\frac{1}{2} \dot{\phi}^{2}-V(\phi)}{\frac{1}{2} \dot{\phi}^{2}+V(\phi)}
$$

and a mass given by

$$
m_{\phi}^{2} \equiv \frac{\partial^{2} V}{\partial \phi^{2}}=n(n+1) M^{2}\left(\frac{M}{\phi}\right)^{2+n} .
$$

Since the potential $V$ in Equation (1) is an inverse power of $\phi$, the evolution of $\phi$ is an increasing function of time, i.e. of the expansion of the universe, while the mass $m(\phi)$ is a decreasing function.

We define the scale factor $a_{c}$ and mass parameter $M$ as the scale when the scalar field takes the value of $M\left(\right.$ i.e. $\left.\phi_{c}\left(a_{c}\right)=M\right)$ and at this time we have 


$$
V\left(\phi\left(a_{c}\right)\right)=M^{4}, \quad m\left(\phi\left(a_{c}\right)\right)=\sqrt{n(n+1)} M .
$$

The parameters $a_{c}, M$ and the DE energy density parameter $\Omega_{\phi} \equiv \rho_{\phi} / \rho_{\text {tot }}$ at $a_{c}$ are related by

$$
\Omega_{\phi c}=\frac{3 M^{4}}{\rho_{\text {mo }} a_{c}^{-3}+\rho_{r o} a_{c}^{-4}+3 M^{4}}
$$

where the initial conditions at $a_{c}$ are $\phi_{c}\left(a_{c}\right)=M, \rho_{\phi}\left(a_{c}\right)=2 M^{4} /\left(1-w_{\phi c}\right)$ $=3 M^{4}, \quad w_{\phi c}=1 / 3$, and $\dot{\phi}\left(a_{c}\right)=\sqrt{2 M^{4}\left(1+w_{\phi c}\right) /\left(1-w_{\phi c}\right)}=2 M^{2}$.

For particles in thermal equilibrium as long as they are relativistic we have $m \ll T$ with $T$ the temperature with an energy density given by $\rho_{\phi}(T \gg m)=$ $\left(\pi^{2} / 30\right) g_{\phi} T^{4}$, with $g_{\phi}=1$ the degrees of freedom of $\phi$, and an EoS $w_{\phi}=1 / 3$. Notice that at $a_{c}$ we have $\rho_{\phi}\left(a_{c}\right) \sim M^{4} \sim T^{4}$, i.e. $m \sim M \sim T$. Therefore, for scales $a<a_{c}$ we have $m<T$ and the evolution of $\phi$ has an EoS $w_{\phi}=1 / 3$ while for $a>a_{c}$ the scalar fields are no longer relativistic $m>T$ and its evolution is determined by Equations ((2) and (3)) with the IPL potential in Equation (1).

The homogeneous background approximation must be refined by considering also the perturbations of the different fluids. We stay in the linear regime where the energy density and other quantities can be decomposed into a homogeneous part (commonly denoted with a bar) and a small position-dependent perturbation. We solve the perturbed equations in the syncrhonous gauge defined by the line element [31]:

$$
\mathrm{d} s^{2}=a^{2}(\tau)\left(-\mathrm{d} \tau^{2}+\left(\delta_{i j}+h_{i j}\right) \mathrm{d} x^{i} \mathrm{~d} x^{j}\right),
$$

where $\mathrm{d} \tau \equiv \mathrm{d} t / a$ denotes the conformal time. The perturbations in the energy density and pressure of the scalar field are given by:

$$
\delta \rho_{\phi}=\frac{\overline{\phi^{\prime}} \delta \phi^{\prime}}{a^{2}}+\frac{\mathrm{d} V}{\mathrm{~d} \phi} \delta \phi, \quad \delta P_{\phi}=\frac{\overline{\phi^{\prime}} \delta \phi^{\prime}}{a^{2}}-\frac{\mathrm{d} V}{\mathrm{~d} \phi} \delta \phi,
$$

where the primes stand for conformal time derivatives. The evolution of $\delta \phi$ in Fourier space is determined by:

$$
\delta \phi^{\prime \prime}+2 \mathcal{H} \delta \phi^{\prime}+\left(k^{2}+a^{2} \frac{\mathrm{d}^{2} V}{\mathrm{~d}^{2} \phi}\right) \delta \phi=-\frac{1}{2} \bar{\phi}^{\prime} h^{\prime}
$$

Here $\mathcal{H} \equiv a^{\prime} / a$ is the conformal expansion rate and $h=\operatorname{Tr}\left(h_{i j}\right)$. The scalar field enters the perturbation equations of the other fluids [31] via $\mathcal{H}$ and through extra source terms proportional to $\delta \phi$ and $\delta \phi^{\prime}$. For example, the CDM overdensities $\delta_{c}$ evolve according to:

$$
\delta_{c}^{\prime \prime}+\mathcal{H} \delta_{c}^{\prime}-\frac{3}{2} \mathcal{H}^{2} \sum \Omega_{i} \delta_{i}\left(3 c_{s, i}^{2}+1\right)=0,
$$

where the sum runs over all the fluids with sound speed $c_{s, i}^{2}=\delta P_{i} / \delta \rho_{i}$.

\section{Constraints}

We explore the parameter space using the CosmoMC [32] and CAMB [33] codes 
properly modified to account for the field background and linear perturbation dynamics described in section 2. In our analysis, we take $n=\frac{1}{2}, \frac{3}{4}, 1$ and find the best fit point for each case varying the physical densities of baryons $\left(\Omega_{b} h^{2}\right)$ and cold dark matter $\left(\Omega_{c} h^{2}\right)$, the optical depth $(\tau)$, the spectral index $\left(n_{s}\right)$, the amplitude of scalar perturbations $\left(A_{s}\right), a_{c}$ and the density parameter of the scalar field at that time $\left(\Omega_{\phi c}\right)$ and $M$, where $\Omega_{r} h^{2}$ and $\Omega_{m} h^{2}$ stand for the densities of radiation (photons and three massless neutrino species) and matter (baryons and CDM), respectively; $\Omega_{\phi o}$ is given by the solution of the system of Equations ((2) and (3)) at present time. Table 1 displays the best-fit values with the corresponding goodness of fit $\left(\chi^{2}\right)$ of some selected parameters from measurements on the CMB temperature anisotropy spectrum [3], BAO [4], and SNeIa [34] data. As a reference, we include the results for the $\Lambda$ CDM model. Notice that the IPL models improve the BAO measurements by $20 \%$ compared to $\Lambda \mathrm{CDM}$ and have an equivalent fit for $\mathrm{CMB}$ and SNIa. This shows that a dynamical DE has a better fit than $\Lambda \mathrm{CDM}$, where the $\mathrm{BAO}$ measurements allow us to constrain the dynamics of the DE.

We see that the transition from the radiation-like state to the scalar-field state takes place well deep in the radiation era for all the cases. However, the transition occurs later as we go to smaller values of $n$, while the scalar field density at $a_{c}\left(\Omega_{\phi c}\right)$ and the scale of energy $M$ increase as we go to larger $n$. The same trend is observed in $H_{o}$ and the DE density parameter at present. Nevertheless, none of these two is larger than in $\Lambda$ CDM.

Table 1. Best fit point for each dark energy model. $H_{o}$ is expressed in $\mathrm{km} \cdot \mathrm{s}^{-1} \mathrm{Mpc}^{-1}$.

\begin{tabular}{|c|c|c|c|c|}
\hline Parameter & $n=1 / 2$ & $n=3 / 4$ & $n=1$ & $\Lambda \mathrm{CDM}$ \\
\hline$a_{c}$ & $1.79 \times 10^{-5}$ & $9.30 \times 10^{-7}$ & $5.48 \times 10^{-8}$ & --- \\
\hline$\Omega_{\phi c}$ & 0.0381 & 0.1479 & 0.2268 & --- \\
\hline$M(\mathrm{eV})$ & 4.63 & 127.31 & 2465.46 & --- \\
\hline$\Omega_{b} h^{2}$ & 0.02225 & 0.02254 & 0.02266 & 0.02242 \\
\hline$\Omega_{c} h^{2}$ & 0.1179 & 0.1174 & 0.1173 & 0.1181 \\
\hline$H_{o}$ & 66.60 & 67.78 & 67.86 & 68.63 \\
\hline$\Omega_{D E o}$ & 0.684 & 0.695 & 0.696 & 0.702 \\
\hline$w_{D E o}$ & -0.906 & -0.932 & -0.924 & -1 \\
\hline$\sigma_{8 o}$ & 0.822 & 0.849 & 0.865 & 0.871 \\
\hline$r_{\mathrm{BAO}}(0.57)$ & 0.0720 & 0.0724 & 0.0752 & 0.07230 \\
\hline$f \sigma_{8}(0.57)$ & 0.4697 & 0.4847 & 0.4934 & 0.5013 \\
\hline$Y_{P}$ & 0.2508 & 0.2628 & 0.2719 & 0.2467 \\
\hline \multicolumn{5}{|c|}{$\chi^{2}(n=1 / 2)=5.737(\mathrm{BAO})+781.811(\mathrm{CMB})+697.300($ SNeIa $)$} \\
\hline \multicolumn{5}{|c|}{$\chi^{2}(n=3 / 4)=5.674(\mathrm{BAO})+776.807(\mathrm{CMB})+695.581($ SNeIa $)$} \\
\hline \multicolumn{5}{|c|}{$\chi^{2}(n=1)=5.589(\mathrm{BAO})+776.214(\mathrm{CMB})+695.658(\mathrm{SNeIa})$} \\
\hline \multicolumn{5}{|c|}{$\chi^{2}(\Lambda \mathrm{CDM})=7.115(\mathrm{BAO})+776.883(\mathrm{CMB})+695.075($ SNeIa $)$} \\
\hline
\end{tabular}


The evolution of the matter, radiation and DE densities is shown in Figure 1. Since our model contains at early times $\left(a<a_{c}\right)$ relativistic particles, its energy density evolves as $\rho_{\phi} \propto a^{-4}$. However, the scalar field quickly dilutes just after the phase transition at $a_{c}$, since the EOS leaps to 1 so that $\rho_{\phi} \propto a^{-6}$ (c.f. Figure 2). Finally, the scalar field remains subdominant until late times, where $\rho_{\phi} \propto a^{-3\left(1+w_{\phi}\right)}$ slightly but noticeably different from a cosmological constant since $w_{\phi} \neq-1$. Notice that at early times (small a), $\rho_{\Lambda} \ll \rho_{r}$ exposing the naturalness and coincidence problems of the $\Lambda \mathrm{CDM}$ model. The evolution of the scalar field EoS is depicted in Figure 2. Initially we have $w_{\phi}=1 / 3$ (dotted line) since the massless fields of the dark group evolve as radiation. Once the transition
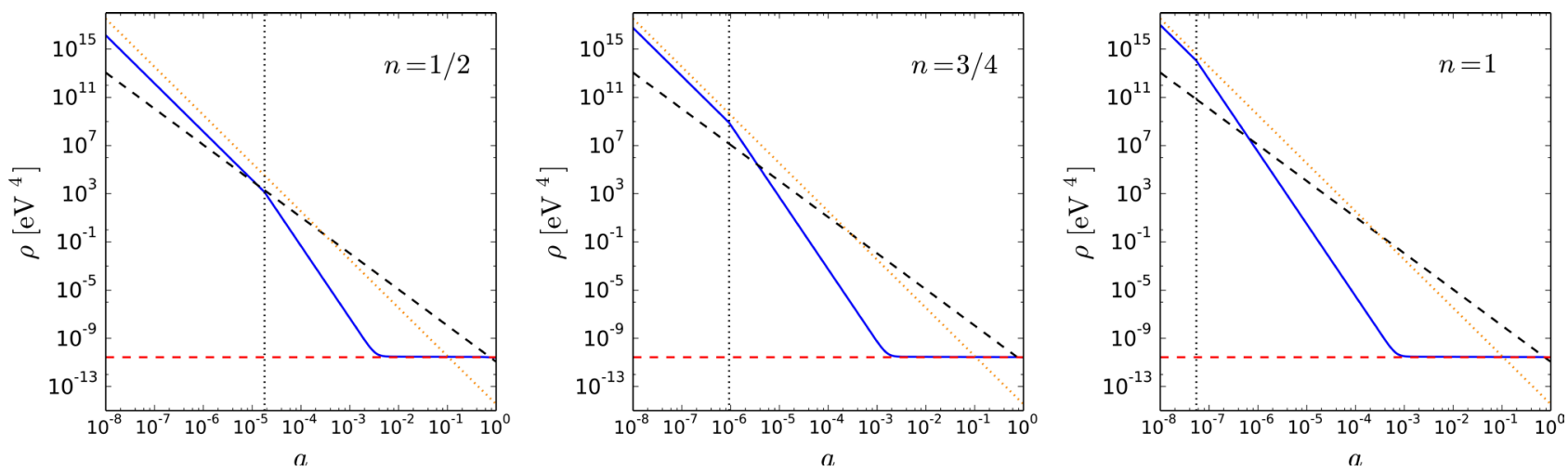

Figure 1. We show the evolution of energy densities $\rho$ as a function of the scale factor a for radiation (dotted orange), dark matter (dashed black), $\Lambda$ (dashed red) and IPL scalar field $\phi$ (solid blue)) and the vertical dotted line marks the transition epoch $a_{c}$. Notice that $\rho_{\Lambda} / \rho_{r} \ll 1$ at early times but not in IPL models.

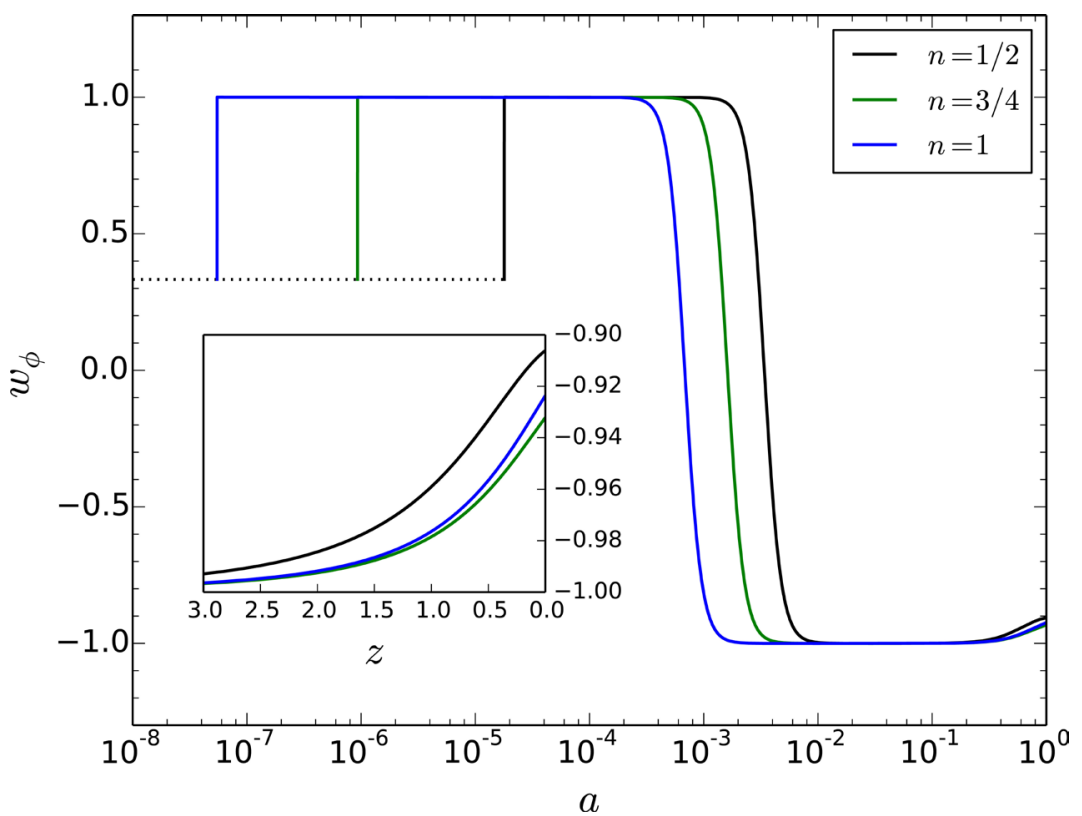

Figure 2. We show the evolution of the $\operatorname{EoS} w_{\phi}$ as afunction of a. Notice the steep transition from $w_{\phi}=1 / 3$ to $w_{\phi}=1$ for $a \ll 10^{-4}$ and a leap to $w_{\phi}=-1$ for $a \sim 10^{-3}$ to finally grow to $w_{\phi}>-1$ at present time for all three cases $n=\frac{1}{2}, \frac{3}{4}, 1$. 
to the scalar field occurs, the EOS leaps abruptly to 1 and stays at this value for some time, then drops to -1 around the decoupling epoch $\left(z_{*} \approx 1090\right.$ for the three models) and finally grows to $w_{D E O}>-1$ at recent times. The inner panel zooms into the late-time behaviour.

It is interesting to note that in [30] an IPL potential with $n=1 / 2$ was also studied but with different initial conditions for $\phi$. In [30] the evolution of the EoS close to present time is a decreasing function from $w=-0.8$ to $w_{o}=-0.87$. They assumed that the potential $V$ has reached the tracking regime [14]. As we can see, different initial conditions have a distinctive evolution of the EoS since in our case we have a growing EoS from $w \simeq-1$ to $w_{o}=-0.906$ at present time (see Table 1). Clearly the choice of initial conditions of $\phi$ is also important.

\section{Cosmological Implications}

\subsection{Distances}

The different late-time dynamics followed by the EOS leaves distinctive imprints on some quantities probed by the cosmological observables. The immediate consequence of such difference is the change in the amount of DE which modifies the size and evolution of the expansion rate $H$ affecting the cosmological distances measured by the SNeIa, BAO and CMB observations. In this regard, we recall that SNeIa flux measurements provide an estimation of the luminosity distance given by $d_{L}(z)=(1+z) \int_{0}^{z} \mathrm{~d} z^{\prime} / H\left(z^{\prime}\right)$, while $\mathrm{BAO}$ measurements are sensitive to the ratio $r_{B A O}(z) \equiv r_{\text {drag }} / D_{V}(z) \quad$ (c.f. Table 1), where $r_{\text {drag }}$ is the coomoving sound horizon at the drag epoch, $D_{V}(z) \equiv\left[(1+z)^{2} D_{A}(z)^{2} z / H(z)\right]^{1 / 3}$ and $D_{A}(z)=(1+z)^{-1} \int_{0}^{z} \mathrm{~d} z / H(z)$ is the angular diameter distance of the reconstructed cluster of galaxies. Figure 3 shows the comoving expansion rate $H(z) /(1+z)$ for the three IPL models and $\Lambda$ CDM. We see that $H(z) /(1+z)$ is larger in IPL in the range $0.4<z<2.3$ sensitive to $\mathrm{BAO}$ and SNIa measurements. Moreover, since the accuracy of the angular size of the sound horizon at recombination $Z_{*} \approx 1090$ obtained from CMB measurements forces IPL and $\Lambda$ CDM to have the same angular distance $D_{A}\left(z_{*}\right)$-differences less than $0.08 \%$-and since the amount of matter is roughly the same-differences less than $0.2 \%$-, the amount of $\rho_{\phi}$ at present time must be reduced from $\rho_{\Lambda}$, giving a smaller $H_{o}^{2}$ in IPL than in $\Lambda \mathrm{CDM}$.

\subsection{CMB and Matter Power Spectrum}

At perturbation level, the IPL model also leaves important imprints on the CMB power spectrum and the evolution of matter perturbations. When we run the models with the same parameters, a change in cosmological distances is reflected in a shift of the position of the peaks of the CMB power spectrum. Moreover, although the amount of extra early radiation vanishes once the transition occurs and therefore it does not have any direct influence on the physical processes from that time onwards, a larger value of primordial helium fraction $Y_{P}$ produced by this extra radiation (c.f. Table 1) leads to a more damped tail of the 
CMB spectrum [35] [36] at high multipoles $l \geq 1000$. On the other hand, the low- $I$ region is sensitive to perturbation dynamics via the late-time ISW effect. In general, models with DE perturbations with a sound speed $c_{s}^{2}>0$ enhance the power [37] [38] [39]. When fitting to the data, some of these effects are compensated or even mimicked by a proper combination of the cosmological

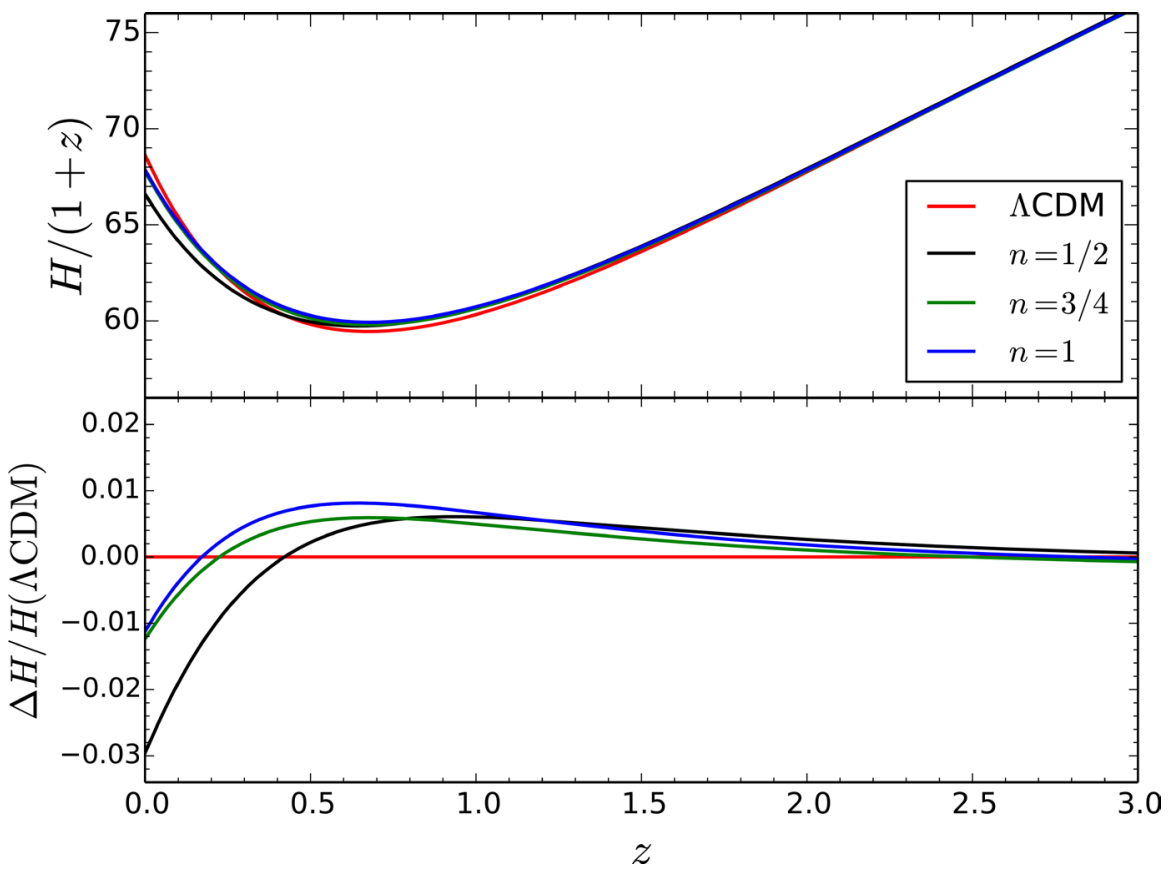

Figure 3. Evolution of $H(z) /(1+z)$ (in $\mathrm{km} \cdot \mathrm{s}^{-1} \cdot \mathrm{Mpc}^{-1}$ ) for IPL models and $\Lambda \mathrm{CDM}$. Lower panel shows the relative differences.

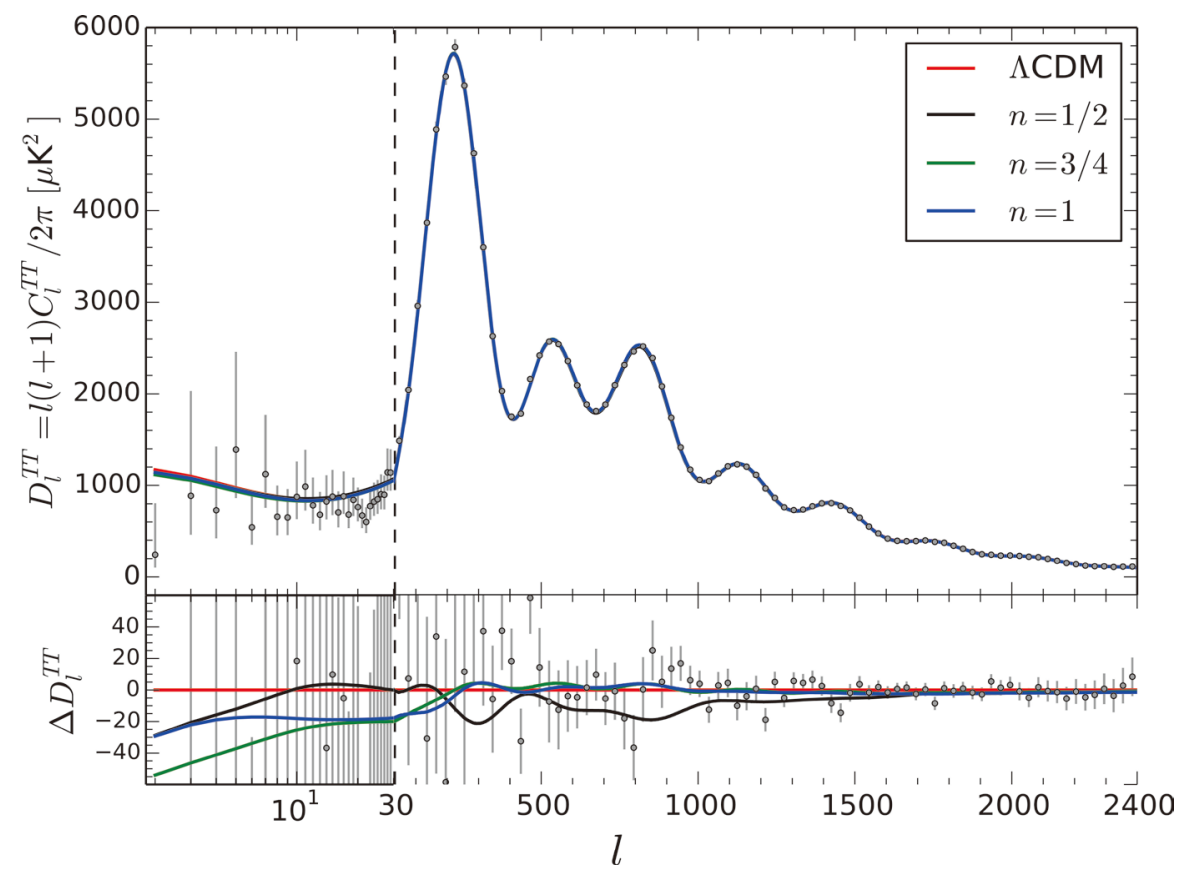

Figure 4. CMB Power Spectrum for IPL models and $\Lambda$ CDM. Lower panel shows the residuals with respect to $\Lambda \mathrm{CDM}$. Grey dots correspond to Planck 2015 measurements [3]. 
parameters. Figure 4 shows the fit of the IPL and $\Lambda$ CDM models to the Planck $2015 \mathrm{CMB}$ temperature anisotropy spectrum. Although the models with $n=3 / 4$ and $n=1$ have a slightly better fit to the data, we note that the amount of early extra radiation introduced by the model with $n=1$ is in unavoidable tension with Big Bang Nucleosynthesis constraints on primordial helium [40] [41] and deuterium [42] [43].

As far as the evolution of matter perturbations is concerned, Figure 5 shows the resulting matter power spectra. In general, the spectrum is suppressed in the IPL models, where the differences with respect to $\Lambda \mathrm{CDM}$ lie within the $6 \%$ in the linear regime $k \lesssim 0.01 \mathrm{Mpc}^{-1}$. Taking the best fit point as input parameters, we can make predictions on quantities that characterize structure formation such as the redshift space distortion (RSD) parameter $f \sigma_{8}$ shown in Figure 6 as a function of $z$. Like the matter power spectrum, the IPL models suppress $f \sigma_{8}$ by nearly a constant fraction in the range $0.6<z<1.2$. Future precision cosmological measurements will allow us to further constraint the dynamical DE models where RSD will play an important role as well as better distance measurements.

\section{Conclusion}

Here we consider three different IPL potentials $V=M^{4+n} \phi^{-n}$ models with a power $n=1 / 2,3 / 4$ and $n=1$. We have seen that they have a better cosmological fit than the standard $\Lambda$ CDM model, reducing BAO $\chi^{2}$ by $20 \%$ and leaving CMB an SNIa with an equivalent fit. With more precise measurements coming in the near future we could be at the stage to determine the dynamics of Dark Energy. In particular BAO and RSD data will be vital to achieve this goal.

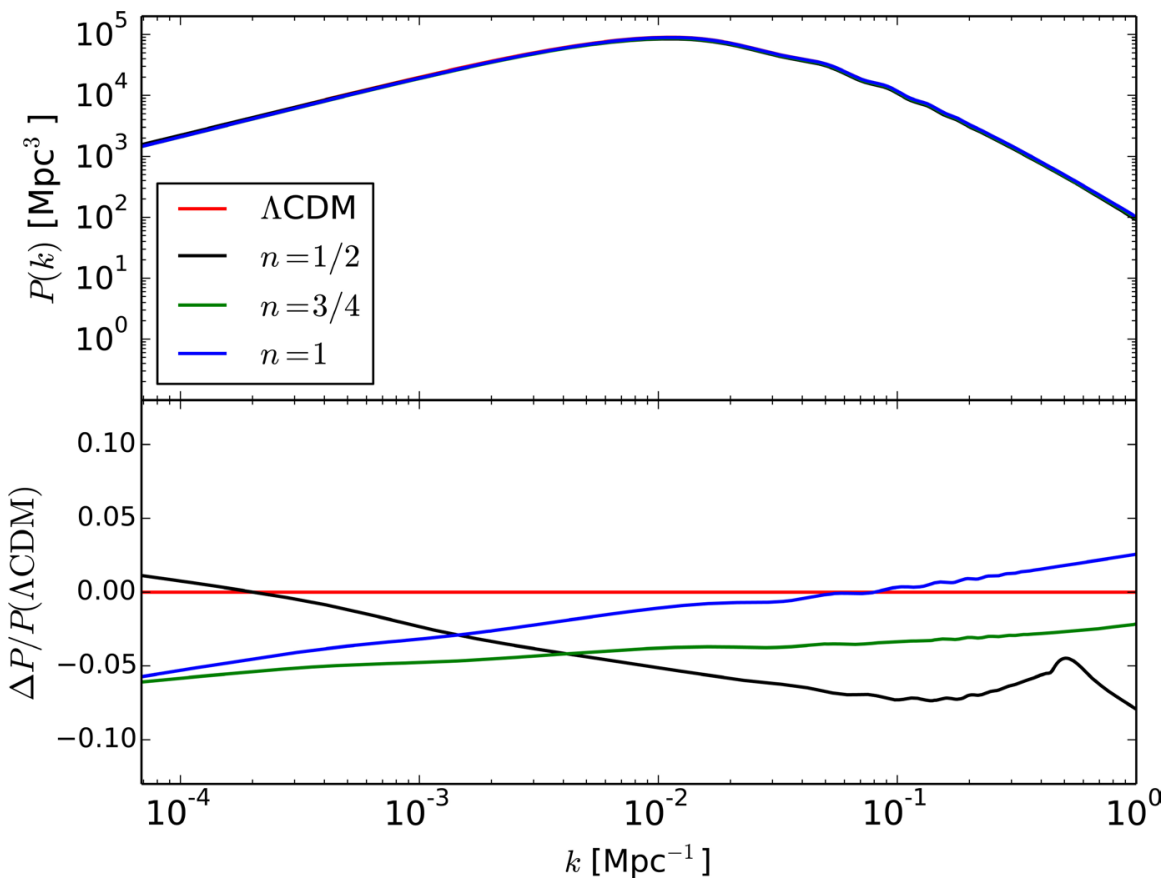

Figure 5. Matter Power Spectrum for IPL models and $\Lambda$ CDM. Lower panel shows the relative differences. 


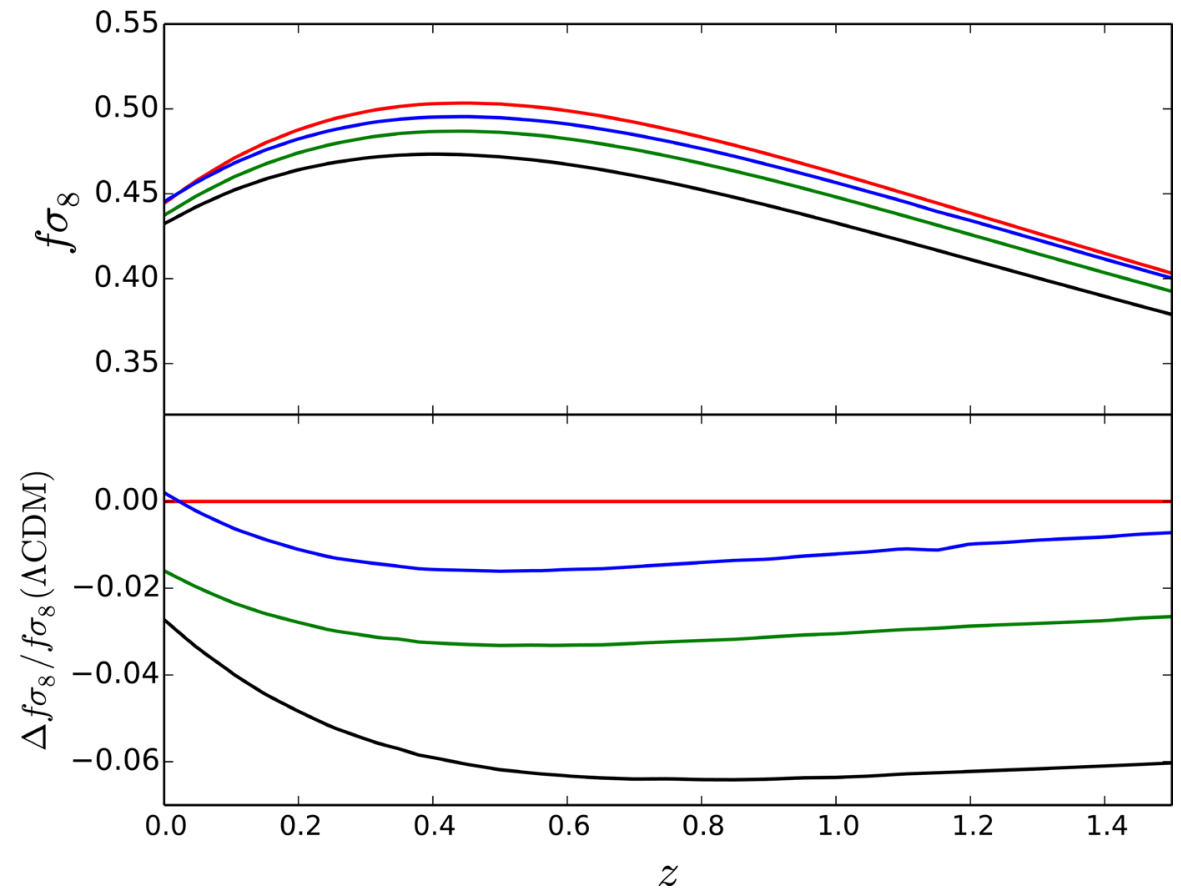

Figure 6. $f \sigma_{8}$ as a function of $z$ for IPL models and $\Lambda$ CDM. Lower panel shows the relative differences.

\section{Acknowledgements}

We acknowledge financial support from UNAM DGAPA IN103518 and Conacyt Fronteras 281.

\section{References}

[1] Riess, A., Macri, L., Casertano, S., Lampeitl, H., Ferguson, H.C., et al. (2011) The Astrophysical Journal, 730, 119. https://doi.org/10.1088/0004-637X/730/2/119

[2] Perlmutter, S., et al. (1999) Measurements of $\Omega$ and $\Lambda$ from 42 High-Redshift Supernovae. Astrophysical Journal, 517, 565. https://doi.org/10.1086/307221

[3] Ade, P.A.R., Aghanim, N., Arnaud, M., Ashdown, M., Aumont, J., Baccigalupi, C., et al. (2016) Astronomy \& Astrophysics, 594, Article No.: A13.

https://doi.org/10.1051/0004-6361/201525830

[4] Gil-Marín, H., et al. (2016) Monthly Notices of the Royal Astronomical Society, 460, 4210-4219. https://doi.org/10.1093/mnras/stw1264

[5] Ross, A., Samushia, L., Howlett, C., Percival, W.J., Burden, A. and Manera, M. (2015) Monthly Notices of the Royal Astronomical Society, 449, 835-847. https://doi.org/10.1093/mnras/stv154

[6] Beutler, F., Blake, C., Colless, M., Jones, D.H., Staveley-Smith, L., et al. (2011) Monthly Notices of the Royal Astronomical Society, 416, 3017-3032.

https://doi.org/10.1111/j.1365-2966.2011.19250.x

[7] http://desi.lbl.gov/

[8] https://www.lsst.org/

[9] http://sci.esa.int/euclid/

[10] Martin, J. (2012) Comptes Rendus Physique, 13, 566-665. https://doi.org/10.1016/j.crhy.2012.04.008 
[11] Tsujikawa, S. (2013) Classical and Quantum Gravity, 30, Article ID: 214003. https://doi.org/10.1088/0264-9381/30/21/214003

[12] Bamba, K., Capozziello, S., Nojiri, S. and Odintsov, S.D. (2012) Astrophysics and Space Science, 342, 155-228. https://doi.org/10.1007/s10509-012-1181-8

[13] Copeland, E.J., Sami, M. and Tsujikawa, S. (2006) International Journal of Modern Physics D, 15, 1753-1936. https://doi.org/10.1142/S021827180600942X

[14] Steinhardt, P.J., Wang, L.M. and Zlatev, I. (1999) Physical Review D, 59, Article ID: 123504. https://doi.org/10.1103/PhysRevD.59.123504

[15] Chen, Y., Geng, C.Q., Cao, S., Huang, Y.M. and Zhu, Z.H. (2015) JCAP, 1502, 010.

[16] Smer-Barreto, V. and Liddle, A.R. (2017) JCAP, 1701, 023.

[17] Intriligator, K.A. and Seiberg, N. (1996) Nuclear Physics B-Proceedings Supplements, 45, 1-28. https://doi.org/10.1016/0920-5632(95)00626-5

[18] Affleck, I., Dine, M. and Seiberg, N. (1985) Nuclear Physics B, 256, 557-599. https://doi.org/10.1016/0550-3213(85)90408-0

[19] Binetruy, P. (1999) Physical Review D, 60, Article ID: 063502. https://doi.org/10.1103/PhysRevD.60.063502

[20] de la Macorra, A. (2003) JHEP, 01, 033.

[21] de la Macorra, A. (2005) Physical Review D, 72, Article ID: 043508. https://doi.org/10.1103/PhysRevD.72.043508

[22] de la Macorra, A. and Stephan-Otto, C. (2001) Physical Review Letters, 87, Article ID: 271301. https://doi.org/10.1103/PhysRevLett.87.271301

[23] de la Macorra, A. and Stephan-Otto, C. (2002) Physical Review D, 65, Article ID: 083520. https://doi.org/10.1103/PhysRevD.65.083520

[24] Ade, P.A.R., et al. (2016) Astronomy \& Astrophysics, 594, A14. https://doi.org/10.1051/0004-6361/201525814

[25] Peebles, P.J.E. and Ratra, B. (1988) APJ, 325, L17. https://doi.org/10.1086/185100

[26] Ratra, B. and Peebles, P.J.E. (1988) Physical Review D, 37, 3406. https://doi.org/10.1103/PhysRevD.37.3406

[27] Wetterich, C. (1988) Nuclear Physics B, 302, 668-696. https://doi.org/10.1016/0550-3213(88)90193-9

[28] Wang, P.Y., Chen, C.W. and Chen, P. (2012) JCAP, 1202, 016.

[29] Ferreira, P.G. and Joyce, M. (1998) Physical Review D, 58, Article ID: 023503. https://doi.org/10.1103/PhysRevD.58.023503

[30] Alimi, J.-M., Fuzfa, A., Boucher, V., Rasera, Y., Courtin, J. and Corasaniti, P.-S. (2010) Monthly Notices of the Royal Astronomical Society, 401, 775-790. https://doi.org/10.1111/j.1365-2966.2009.15712.x

[31] Ma, C. and Bertschinger, E. (1995) The Astrophysical Journal, 455, 7-25. https://doi.org/10.1086/176550

[32] Lewis, A. and Bridle, S. (2002) Physical Review D, 66, Article ID: 103511. https://doi.org/10.1103/PhysRevD.66.103511

[33] Lewis, A., Challinor, A. and Lasenby, A. (2000) The Astrophysical Journal, 538, 473-476. https://doi.org/10.1086/309179

[34] Betoule, M., et al. (2014) Astronomy \& Astrophysics, 568, Article No.: A22.

[35] Hu, W. and White, M. (1997) APJ, 479, 568-579. https://doi.org/10.1086/303928

[36] Hou, Z., Keisler, R., Knox, L., Millea, M. and Reichardt, C. (2013) Physical Review $D, \mathbf{8 7}$, Article ID: 083008. https://doi.org/10.1103/PhysRevD.87.083008 
[37] Bean, R. and Dore, O. (2004) Physical Review D, 69, Article ID: 083503. https://doi.org/10.1103/PhysRevD.69.083503

[38] Weller, J. and Lewis, A.M. (2003) Monthly Notices of the Royal Astronomical Society, 346, 987-993. https://doi.org/10.1111/j.1365-2966.2003.07144.x

[39] Cabass, G., Gerbino, M., Giusarma, E., Melchiorri, A., Pagano, L. and Salvati, L. (2015) Physical Review D, 92, Article ID: 063534. https://doi.org/10.1103/PhysRevD.92.063534

[40] Aver, E., Olive, K., Porter, R.L. and Skillman, E. (2013) JCAP, 11, 017.

[41] Izotov, Y., Thuan, T. and Guseva, N. (2014) Monthly Notices of the Royal Astronomical Society, 445, 778-793. https://doi.org/10.1093/mnras/stu1771

[42] Iocco, F., Mangano, G., Miele, G., Pisanti, O. and Serpico, P.D. (2009) Physics Reports, 472, 1-76. https://doi.org/10.1016/j.physrep.2009.02.002

[43] Riemer-Sørensen, S., et al. (2015) Monthly Notices of the Royal Astronomical Society, 447, 2925-2936. https://doi.org/10.1093/mnras/stu2599 